\title{
Creation and Maintenance of a Living Biobank - How We Do It
}

\author{
Florian Bürtin ${ }^{1}$, Stephanie Matschos ${ }^{2}$, Friedrich Prall ${ }^{3}$, Christina S. Mullins ${ }^{2}$, Mathias Krohn ${ }^{2}$, Michael Linnebacher $^{2}$ \\ ${ }^{1}$ Department of General, Visceral, Vascular and Transplantation Surgery, University Medical Center Rostock, University of Rostock ${ }^{2}$ Molecular Oncology \\ and Immunotherapy, Department of General, Visceral, Vascular and Transplantation Surgery, University Medical Center Rostock ${ }^{3}$ Institute of Pathology, \\ University Medical Center Rostock
}

\section{Corresponding Author}

Michael Linnebacher

michael.linnebacher@med.uni-rostock.de

\section{Citation}

Bürtin, F., Matschos, S., Prall, F.,

Mullins, C.S., Krohn, M.,

Linnebacher, M. Creation and

Maintenance of a Living Biobank - How

We Do It. J. Vis. Exp. (170), e62065,

doi:10.3791/62065 (2021).

\section{Date Published}

April 10, 2021

DOI

$10.3791 / 62065$

URL

jove.com/video/62065

\section{Abstract}

In light of the growing knowledge about the inter-individual properties and heterogeneity of cancers, the emerging field of personalized medicine requires a platform for preclinical research. Over recent years, we have established a biobank of colorectal and pancreatic cancers comprising of primary tumor tissue, normal tissue, sera, isolated peripheral blood lymphocytes (PBL), patient-derived xenografts (PDX), as well as primary and secondary cancer cell lines. Since original tumor tissue is limited and the establishment rate of primary cancer cell lines is still relatively low, PDX allow not only the preservation and extension of the biobank but also the generation of secondary cancer cell lines. Moreover, PDX-models have been proven to be the ideal in vivo model for preclinical drug testing. However, biobanking requires careful preparation, strict guidelines and a well attuned infrastructure. Colectomy, duodenopancreatectomy or resected metastases specimens are collected immediately after resection and transferred to the pathology department. Respecting priority of an unbiased histopathological report, at the discretion of the attending pathologist who carries out the dissections, small tumor pieces and non-tumor tissue are harvested.

Necrotic parts are discarded and the remaining tumor tissue is cut into small, identical cubes and cryopreserved for later use. Additionally, a small portion of the tumor is minced and strained for primary cancer cell culture. Additionally, blood samples drawn from the patient pre- and postoperatively, are processed to obtain serum and PBLs. For PDX engraftment, the cryopreserved specimens are defrosted and implanted subcutaneously into the flanks of immunodeficient mice. The resulting PDX closely recapitulate the histology of the "donor" tumors and can be either used for subsequent xenografting or cryopreserved for later use. In the following work, we describe the individual steps of creation, maintenance and administration of a large 
biobank of colorectal and pancreatic cancer. Moreover, we highlight the crucial details and caveats associated with biobanking.

\section{Introduction}

In recent years, the accumulated knowledge of cancers' morphologic, clinical and genetic properties led to the conception of cancer as a heterogeneous, individual disease. Consequently, mutational characterization of neoplasms, besides clinical and pathological features, has gained importance for clinical decision making and many targeted therapies were developed for various molecular alterations. For instance, the efficacy of cetuximab in colorectal cancer treatment can be predicted by the analysis of the KRAS and PIK3CA mutational status ${ }^{1}$. Precision medicine aims for a tailored approach to provide the highest treatment response in each patient and avoid toxicity of inefficacious therapies ${ }^{2}$. Biobanks contain tissue, blood and other biological materials of cancer patients, which are linked to the clinical data, and thus are an excellent tool for translational cancer research. Due to the large number of clinical samples, biobanks enable the detection of rare, but potentially druggable mutations, which provides new treatment opportunities for the individual patient $^{3}$.

To cover as broad as possible an oncologic research spectrum, we did not restrain our activity on sample harvesting alone, but focused on the establishment of patientderived cancer cell lines and xenografts (PDX). Traditional
2D cell lines remain the corner stone of in vitro research and are the prime choice for large scale drug screenings ${ }^{4,5}$. Moreover, cell line analysis is often easier, cheaper and more readily available. Additionally, since patient-derived peripheral blood lymphocytes (PBL) are available, also tumor immunology can be studied in vitro ${ }^{6}$. However, the majority of newly developed drugs with promising preclinical effectivity in cell based in vitro or in vivo experiments, have shown disappointing results in clinical trials ${ }^{7}$. In contrast, preclinical studies based on PDX in vivo studies have reflected the clinical activity of antineoplastic agents much more faithfully ${ }^{8}$. Since PDX tissue closely reflects the histological and molecular properties of the donor tumor, PDX models are a good way to propagate the often very limited amounts of viable tumor tissue to maintain the integrity of a biobank and to allow the exchange of samples between research groups and institutions. Moreover, cancer cell lines derived from PDX tissue can be established significantly easier than primary cancer cell lines ${ }^{9}$. In recent years, our working group has established a comprehensive integrated colorectal and pancreatic cancer biobank by stepwise standardizing and optimizing the work flow for all biological samples in question (Figure 1). 


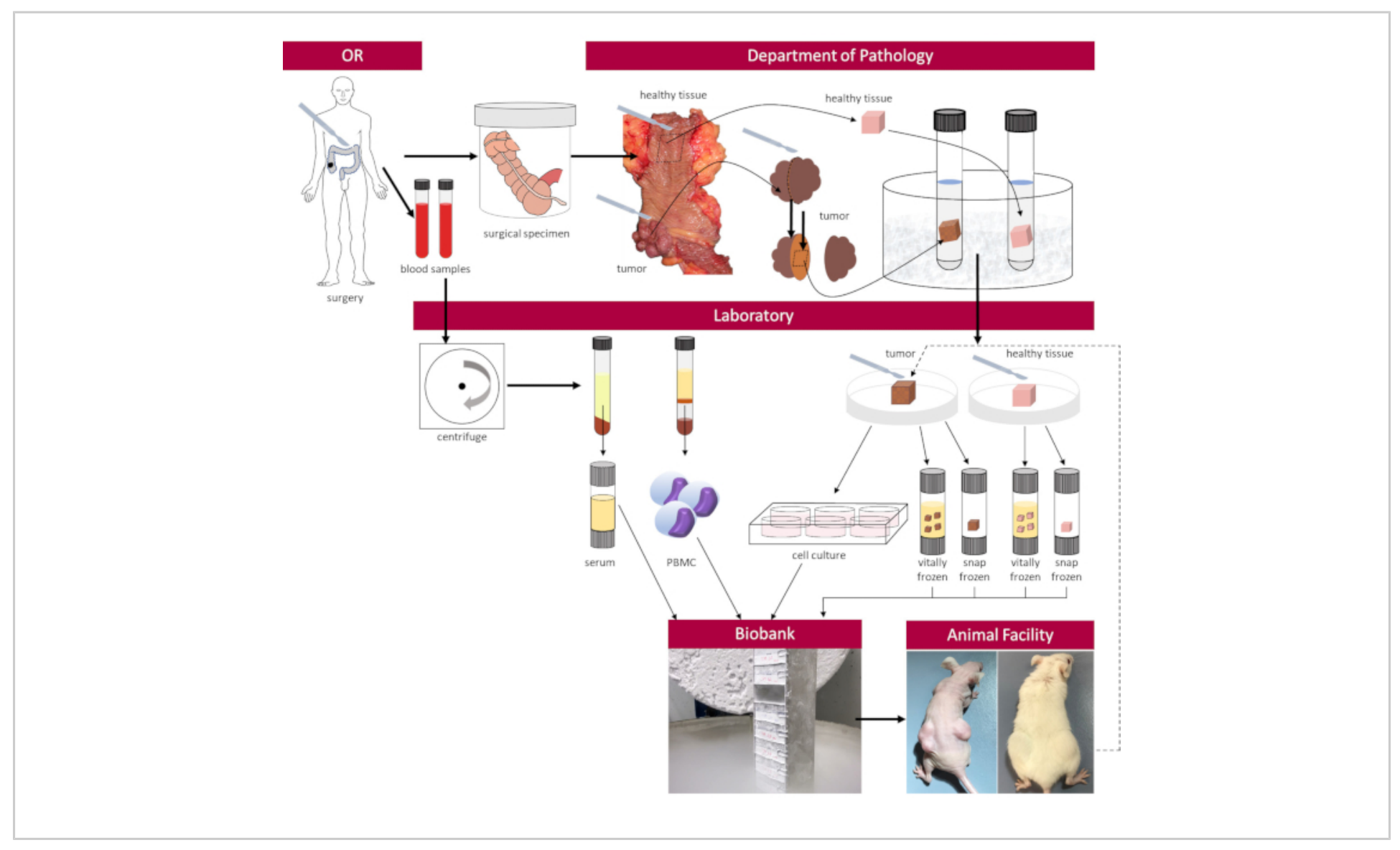

Figure 1: Workflow and organization of the biobank Please click here to view a larger version of this figure.

\section{Protocol}

The following study has been approved by the institutional review board of the University Medical Center Rostock (II HV 43/2004, A 45/2007, A 2018-0054, A 2019-0187 and A 2019-0222). Furthermore, all veterinary relevant procedures have been approved by the Landesamt für Landwirtschaft, Lebensmittelsicherheit und Fischerei Mecklenburg-Vorpommern under the registration numbers LALLF M-V/TSD/ 7221.3-2-020/17 and 7221.3-1-007/19.

\section{Experimental Prerequisites}

1. Meet several important framework conditions to establish and maintain a biobank.

1. Use a clinic with a surgical department and sufficient number of oncological resections together with a well-equipped lab and sufficient academic staff. A good infrastructure and a firm liaison with a cooperating pathology department are further prerequisites.

2. For in vivo research, use an animal facility with housing conditions appropriate to immunodeficient mice. 
3. Obtain authorization on any research on patientderived material from a health care ethics committee. Obtain approval on any in vivo research from the competent authority according to local statutory regulations.

\section{Sample collection}

1. The day before surgery

1. Evaluate all patients with resectable colorectal or pancreatic cancer and/or corresponding metastases for biobanking eligibility. Avoid including cases with neoadjuvant pretreatment, very small tumors, tumors of uncertain dignity or lesions which have been partly resected endoscopically before.

2. Obtain written approval of participation from the patient during the informed consent discussion about the surgical procedure. Inform timely all involved surgeons, the laboratory team as well as the pathologist.

2. Sample acquisition

1. Inform all attendants in the operation room (OR) about the tissue collection for the biobank immediately before the start of the surgical procedure.

NOTE: It is crucial that the tissue must not be fixed in formalin. If the tissue is submerged in formalin, it becomes unsuitable for integrated biobanking.

2. Draw $40 \mathrm{~mL}$ of heparinized blood $(2 \times 20 \mathrm{~mL}$ syringe) as well as a standard $7.5 \mathrm{~mL}$ serum tube immediately after anesthetic induction and transfer quickly to the lab for PBL isolation and serum processing (see step 3-4).
3. Obtain the resected specimen directly from the operating table, place it into an appropriate container and take it to the pathological department. Write down the time point of detachment from the circulation, resection and arrival at pathology.

NOTE: The suitability of the specimen for bio banking should be assessed by the cooperating pathologist who dissects a slice of tumor and nonmalignant tissue. Do not excise any parts of the specimen by yourself which may compromise the subsequent pathological report.

4. Place both tissue pieces in a separate 15 to $50 \mathrm{~mL}$ polypropylene tube with 10 to $30 \mathrm{~mL}$ tissue storage solution (or DPBS) on ice. Write down the time of receipt and transfer the specimens immediately to the lab.

NOTE: The following protocol steps 3-6 must be conducted in a laminar flow cabinet under strict sterile conditions. Use all liquids at room temperature.

\section{Serum processing}

1. Centrifuge the $7.5 \mathrm{~mL}$ serum tube at $1128 \times \mathrm{g}$ and $4{ }^{\circ} \mathrm{C}$ for $15 \mathrm{~min}$ in a pre-cooled centrifuge.

2. Aliquot $1 \mathrm{~mL}$ serum per tube in pre-labeled cryotubes and freeze in liquid nitrogen.

\section{Isolation of PBL by density gradient centrifugation}

NOTE: Work parallel with each of the two $20 \mathrm{~mL}$ syringes.

1. Fill $20 \mathrm{~mL}$ of heparinized blood into a $50 \mathrm{~mL}$ polypropylene tube and add $15 \mathrm{~mL}$ of DPBS. 
2. Take $15 \mathrm{~mL}$ of Pancoll with a serological pipette, insert the pipette carefully all the way to the bottom of the polypropylene tube and release the Pancoll very slowly to form a layer beneath the blood/DBPS column.

3. Centrifuge at $375 \times \mathrm{g}$ for $15 \mathrm{~min}$ without brake.

4. Aspirate and transfer the opaque interphase layer between the mid and top column of both samples into a fresh $50 \mathrm{~mL}$ Polypropylene tube and fill up with DPBS to $50 \mathrm{~mL}$.

5. Centrifuge at $270 \times \mathrm{g}$ for $15 \mathrm{~min}$ with brake.

6. Aspirate and discard the supernatant, resuspend the cell pellet in $4.5 \mathrm{~mL}$ of freezer medium.

7. Aliquot $1.5 \mathrm{~mL}$ of the suspension per cryotube, close the tubes tightly and place them in a freezing container suitable for slow freezing and store at $-80{ }^{\circ} \mathrm{C}$.

\section{Tissue processing}

NOTE: Start with the generation of snap frozen samples of tumor and healthy tissue to maintain the integrity of nucleic acids.

1. Tumor tissue specimen

1. Transfer the tumor specimen with several $\mathrm{mL}$ of tissue storage solution from the polypropylene tube to a Petri dish. Rinse with DPBS if necessary. Avoid touching the specimen and use two sterile scalpels to handle the tissue. Avoid desiccation at any time.

2. Weigh the tumor specimen on a convenient scale in a separate dish and note the tissue weight.

3. Evaluate size, shape and tissue quality of the tumor tissue before cutting. Aim to obtain at least one piece about the size of a pinhead for snap freezing and four cubes of approx. $30 \mathrm{~mm}^{3}$ (edge length $3 \mathrm{x}$ $3 \times 3 \mathrm{~mm}$ ) each for vital cryopreservation. Generate as many $30 \mathrm{~mm}^{3}$-cubes in quadruples as possible and generate one piece for snap freezing per 5 quadruples. Also take into account that necrotic portions must be cut off so that the cubes consist of vital tissue only.

4. Generate slices of $3 \mathrm{~mm}$ thickness. Cut off necrotic portions, distinguishable as gel-like or liquid mass, and cut the slices into cubes of the two desired sizes.

\section{NOTE: Do not discard any tissue at this point.}

5. Snap freezing

1. Label cryotubes accordingly (see step 7.7).

2. Place one small tissue piece per pre-labeled cryotube. Submerge the samples immediately into liquid nitrogen for several minutes and store at $-80{ }^{\circ} \mathrm{C}$ subsequently.

6. Cryopreservation of vital tissue

1. Label cryotubes accordingly (see step 7.7) and fill each with $1.5 \mathrm{~mL}$ of freezer medium. Place the freezing container beside the bench.

NOTE: Follow the next steps as quickly as possible. Since the DMSO in the freezer medium has cytotoxic properties, the time of the tissue being submerged in freezer medium without proper cooling should not exceed 2 minutes.

2. Arrange the $30 \mathrm{~mm}^{3}$ cubes in quadruples. Shove necrotic tissue and other remains to the edge of the dish, but do not discard them.

3. Scoop the cubes with the scalpel blade and transfer 4 cubes per cryotube. Make sure that 
the tumor pieces are entirely submerged in freezer medium. Close the tubes tightly and place them in a freezing container suitable for slow freezing and store in $\mathrm{a}-80^{\circ} \mathrm{C}$ freezer.

4. Transfer cryotubes into a suited storage system for long term storage at $-140{ }^{\circ} \mathrm{C}$ or lower. Documentation in the laboratory inventory management system is mandatory.

2. Healthy tissue specimen: Repeat steps 5.1.1. to 5.1.6.4 for the healthy tissue specimen.

\section{Primary cell culture}

1. Disintegrate the remains of the tumor tissue, including the necrotic scrap, in the Petri dish with the scalpels to pieces as small as possible.

2. Place a sterile cell strainer ( $100 \mu \mathrm{m}$ pore size $)$ on top of a $50 \mathrm{~mL}$ polypropylene tube.

3. Use a serological pipette to add $5-10 \mathrm{~mL}$ of DPBS to the Petri dish, float the tissue remains and pipette up and down to generate a suspension.

4. Transfer the suspension with the pipette to the cell strainer.

5. Repeat Steps 6.3-6.4 until all tissue remains are resolved from the Petri dish.

6. Use the plunger of a $20 \mathrm{~mL}$ one-way syringe to squeeze the cell and tissue suspension through the cell strainer.

7. Rinse with $5-10 \mathrm{~mL}$ of fresh DPBS, discard the cell strainer and close the tube properly.

8. Centrifuge the suspension at $180 \mathrm{xg}$ for $5-10$ minutes.

9. Prepare a collagen-I precoated 6 well plate with $1.5 \mathrm{~mL}$ of medium per well.
10. Aspirate and discard the supernatant. Resuspend the pellet in $3 \mathrm{~mL}$ of DPBS or medium and add $500 \mu \mathrm{L}$ of the suspension to each well. Place the plate into the incubator $\left(100 \%\right.$ humidity, $\left.5 \% \mathrm{CO}_{2}, 37^{\circ} \mathrm{C}\right)$

11. Monitor the plate daily for cell growth and contamination. NOTE: Further cell culturing up to the point of establishment of a permanent cell line is not described here.

\section{PDX Generation}

1. Conduct in vivo experiments only by appropriately qualified persons meeting the requirements of the competent authority of your jurisdiction.

2. House immunodeficient mice under specific-pathogenfree (SPF) conditions satisfying the demands of the used mouse strain. The hygienic measures include individually ventilated cages, autoclaved food, water and nesting material as well as a safety air lock and the wearing of personal, protective equipment.

3. Autoclave all instruments beforehand and use only one set of instruments for each tumor case to avoid crosscontamination. Handle the tumor tissue as aseptic as possible. All plastic items named below should be sterile, single-use and discarded after each surgery.

NOTE: Determined by the method of freezing four tumor tissue pieces per cryotube, the PDX generation requires always two mice per sample, ideally resulting in four PDX tumors.

4. Choose the desired primary tumor for engraftment via the laboratory inventory management system and transfer the sample (vitally preserved tumor tissue) from the main storage tank to a portable liquid nitrogen container 
(Intermediate storage at $-80{ }^{\circ} \mathrm{C}$ on dry ice is also convenient).

5. Put on personal protective equipment before entering the SPF-section (scrubs, clogs, apron, hair cover, surgical mask and overshoes), disinfect your hands and all equipment.

6. Matrigel soaking

1. Remove the cryotube form the liquid nitrogen container and await thawing of the specimen.

2. Label a $50 \mathrm{~mL}$ polypropylene tube and fill with $35 \mathrm{~mL}$ of DPBS.

3. Tilt the cryotube up and down and transfer the content immediately to the polypropylene tube as soon as the tissue-medium-slush can be shifted. Gently rinse the tumor tissue pieces, discard the main volume from the tube in a separate vessel, close the lid and put the tube up-side-down, so that the four tissue pieces gather in the lid.

4. Put a Petri dish on the cooling accumulator and place $100 \mu \mathrm{L}$ of Matrigel as a single droplet into the middle. Use anatomical forceps to transfer the tumor pieces into the Matrigel. Make sure that each piece is covered completely with Matrigel. Incubate for 10 minutes at $4{ }^{\circ} \mathrm{C}$.

7. Mouse anesthesia (2 mice per sample, work in parallel)

1. Prepare a $3: 1$ - stock of a ketamine $(100 \mathrm{mg} / \mathrm{mL})$ and xylazine $(20 \mathrm{mg} / \mathrm{mL})$ anesthetic solution. The recommended dose is $90 / 6 \mathrm{mg} / \mathrm{kg}$ body weight.

2. Weigh the mouse and draw up the necessary anesthetic solution into a single use insulin syringe.

3. Place the mouse on the grid of the cage, pull its tail gently with one hand to induce a forward movement and simultaneously crab the neck with a pinch grip of the other hand. Lift the mouse of the grid and turn the holding hand, so that animal's back rests on your palm. Immobilize one of the hind legs with your pinky and inject the narcotics intraperitoneally. Put the mouse back to its cage and await narcotic induction.

4. Place the anesthetized mouse on the heating plate and cover the eyes with ointment to avoid corneal harm. Asses the depth of anesthesia by gently pinching the back foot of the mouse with surgical forceps.

NOTE: Absence of movement indicates deep narcosis. Any kind of movement either requires more time for reaching the desired narcotic depth or an additional dose of anesthetics.

8. Surgical procedure

1. Form a skin fold by pinching the neck of the mouse and inject the microchip subcutaneously with the applicator (See step 9 for programming details)

2. Shave the flanks of the mouse if necessary (NMRI ${ }^{\text {nu/ }}$ nu mice do not require shaving), apply povidoneiodine with a cotton swab and use surgical drape to create a sterile field.

3. Lift the skin of the flank with surgical forceps, make a small incision of circa $4 \mathrm{~mm}$ and form a small subcutaneous pocket by blunt preparation with scissors.

4. Put one tumor piece into each pocket and place it at the rear end. 
5. Clip the end of a $100 \mu \mathrm{L}$ pipette tip and aspirate the remaining Matrigel from the Petri dish and apply it equally into each skin pocket.

6. Close the wounds with simple interrupted sutures and apply spray dressing.

9. Scan the microchip and check validity of mouse- and tumor-ID.

10. Prepare a new cage with fresh bedding and nesting material, as well as a gnawing stick. Fold a "cushion" out of paper towels and lay down the mouse with elevated head under an infrared heat lamp.

11. Mix $0.25 \mathrm{~mL}$ of trimethoprim/sulfamethoxazole $(400$ $\mathrm{mg} / 80 \mathrm{mg}$ ) with $100 \mathrm{~mL}$ of drinking water and administer via the drinking bottle. Consider that one mouse consumes approximately $150 \mathrm{~mL}$ per $\mathrm{kg}$ body weight daily.

NOTE: Since the subcutaneous PDX-model is not associated with postoperative pain, neither during the wound healing process, nor during tumor outgrowth, postoperative analgesia is not required. Please note that the animal welfare guidelines of your institution/authority may differ.

12. Monitoring of experimental animals

1. Monitor the mice daily for signs of distress. This can be delegated to qualified animal caretakers.

2. Keep up the postoperative antibiotic treatment with the aforementioned dosage for 4 weeks. Replace the antibiotic mixture twice per week.

3. Measure the tumor size at least once per week, ideally daily, with a caliper (tumor volume $=0.52$ $x$ length $x$ width $x$ height $\left[\mathrm{mm}^{3}\right]$ ) and record in the database.

\section{PDX harvesting and processing}

1. Harvest and process the PDX tumor, when:

The tumor size reaches the target volume of $1.500 \mathrm{~mm}^{3}$. The tumor bearing animal shows signs of distress and/or disease and treatment is futile.

The tumor becomes ulcerated or penetrates the skin of the mouse.

2. Read out the microchip to identify the correct PDX.

3. Euthanize the mouse by a legal method (depending on national guidelines) as for example $\mathrm{CO}_{2}$-asphyxation or ketamine/xylazine injection followed by cervical dislocation.

4. Lift the skin with surgical forceps at the flanks and incise with Metzenbaum scissors a few millimeters distant from the tumor.

5. Detach the skin above the tumor by blunt preparation, then carefully grasp the tumor with anatomical forceps and detach the tumor from the superficial fascia of the body.

6. Rinse the tumor with DPBS, put it into a Petri dish and remove adjacent connective tissue.

7. At this point, perform one of the following:

1. Cut $30 \mathrm{~mm}^{3}$ cubes and create new PDX (Proceed with protocol at point 7.7.4).

2. Cut the tumor into slices, which are then transferred to histology cassettes and preserved in $4 \%$ formaldehyde for later paraffin embedding.

3. Preserve the tumor in a tube with tissue storage solution to add it to the biobank (Proceed with protocol at step 3.) and/or create PDX-derived cell lines (Proceed with protocol at step 4.) 


\section{Biobank and data management}

1. Assign an internal ID to each tumor case according to

Table 1.

\begin{tabular}{|c|c|c|c|c|}
\hline $\begin{array}{c}\text { Laboratory } \\
\text { location/name }\end{array}$ & cancer entity & $\begin{array}{c}\text { consecutive } \\
\text { case number }\end{array}$ & specification & consecutive number \\
\hline \multirow{2}{*}{} & $\mathrm{C}=$ colorectal & & _Met=Metastasis & \\
\cline { 2 - 2 } & $\mathrm{P}=$ pancreatic & & _Tu=Tumor & \\
\hline
\end{tabular}

Example: HROC389_Met2 = Rostock, colorectal cancer, case 389, second metastasis

Table 1: Definition of the sample ID.

2. Store the patient consent in electronic and paper form together with the tumor-ID.

3. Gather as much clinical data as possible and store them anonymized and separately.

4. Use a data management software (e.g., Freezerworks) or other and create an interface with a label printing software to generate temperature-resistant, self-sticking bar code labels.

5. Add a new sample by opening the data management software, define the specimen type and record the following information: tumor ID, tissue type, freezing method, date, responsible employee, passage number, mouse ID and mouse strain.

6. Assign the samples to specific positions in the storage tank.

7. Tracing and monitoring of PDX (Applies to step 7-8)

1. Use a MS Access data base (or a similar system) on a portable, Bluetooth-enabled device (laptop or tablet) to record tumor ID, date of implantation, date of euthanasia, mouse age and strain as well as tumor growth over time.

2. Connect the microchip reader to the device and read out the microchip prior to implantation.

3. Assign a specific ID to each mouse; we use the following scheme: (see Table 2 below)

4. After implantation, record the ID together with the mouse characteristics in the data base.

5. Re-read the microchip and check, if the specifications of the microchip, the data base and the cryotube label are consistent.

6. Create a label for each mouse cage accordingly.

NOTE: To create a physical back up, stick the cryotube labels with the corresponding microchip labels into a booklet and note date and mouse strain.

7. To monitor the tumor growth of the individual PDX, scan the microchip of the mouse with the reader connected to the data base device for identification and record the tumor size measured by caliper each week. 
8. Plan the ideal time point of PDX harvesting by analyzing the growth curve of the tumor.

\begin{tabular}{|l|c|c|c|}
\hline Tumor-ID & Prior storage in $\mathrm{N}_{2}(=\mathrm{f})$ & Passage $(=\mathrm{T})$ number & $\begin{array}{c}\text { consecutive mouse } \\
(=\mathrm{M}) \text { number }\end{array}$ \\
\hline
\end{tabular}

Table 2: Definition of the PDX ID.

\section{Representative Results}

In our hands, the establishment rate of primary cell cultures (Figure 2A \& B) was $12.9 \%$ in a large series ${ }^{9}$. The majority of attempts to isolate expandable tumor cells from fresh surgical resected specimens failed due to a lack of outgrowth or early contamination. Cell line establishment was considered successful after 3 passages with a steady growth under standard culture conditions (DMEM, 10\% FCS, standard culture vessel) and validation of epithelial differentiation via FACS-analysis ${ }^{10}$. Cell lines derived from PDX tumors (Figure 2C \& D) showed a higher establishment rate of $23.6 \%$ which is also due to the possibility of repetitive attempts in contrast to primary resected tumors ${ }^{9}$. However, some mixed cultures (Figure 2E) cannot be freed of fibroblastic growth or are even lost due to fibroblastic overgrowth (Figure 2F). 

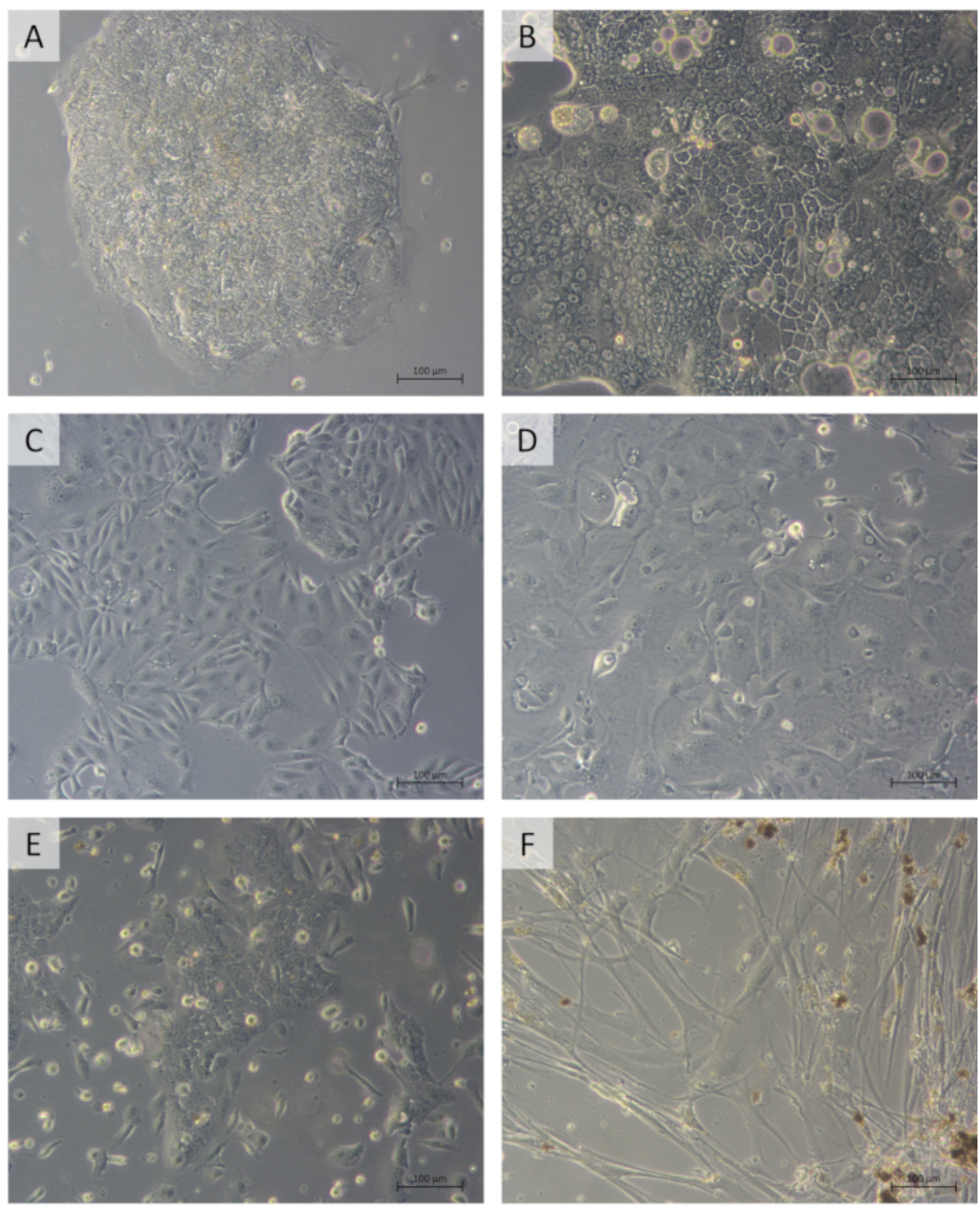

Figure 2: Cell culture. Primary cancer cell lines, derived from a metastasis of colon cancer case HROC313, passage 21 (A) and pancreatic cancer case HROP88, passage 5 (B). PDX-derived cancer cell lines of colon PDX HROC285 T0 M2 (D) and pancreatic PDX HROP10 T5 M2, passage 4 (E). Mixed culture of fibroblasts and cancer cells from pancreatic cancer HROP75, passage $8(C)$ and fibroblastic overgrowth $(F)$. Please click here to view a larger version of this figure.

Considering changes in PDX generation protocol, mouse strains used and also experimenters over several years, as well as large differences in the amount of tumor tissue available for engraftment, it is not trivial to give the overall success rate of PDX generation. In a very recent series of PDX generation experiments performed by two researchers
(S.M. and F.B.), primary outgrowth rates of $63 \%$ for colorectal PDX (an exemplary histology can be depicted from Figure 3A) and $48 \%$ for pancreatic PDX (Figure 3B) were observed. The outgrowth of murine or human lymphomas at the implantation site is relatively rare, but can mimic successful PDX outgrowth (Figure 3C). Apart from histopathological 
examination, concordance between PDX models and their donor patients was regularly confirmed by short tandem repeat (STR) analysis (Figure 3D). To the present day the biobank comprises $>50$ primary and $>50$ secondary colorectal, 3 primary and 6 secondary pancreatic cancer cell lines as well as $>150$ colorectal and 19 pancreatic PDX models.
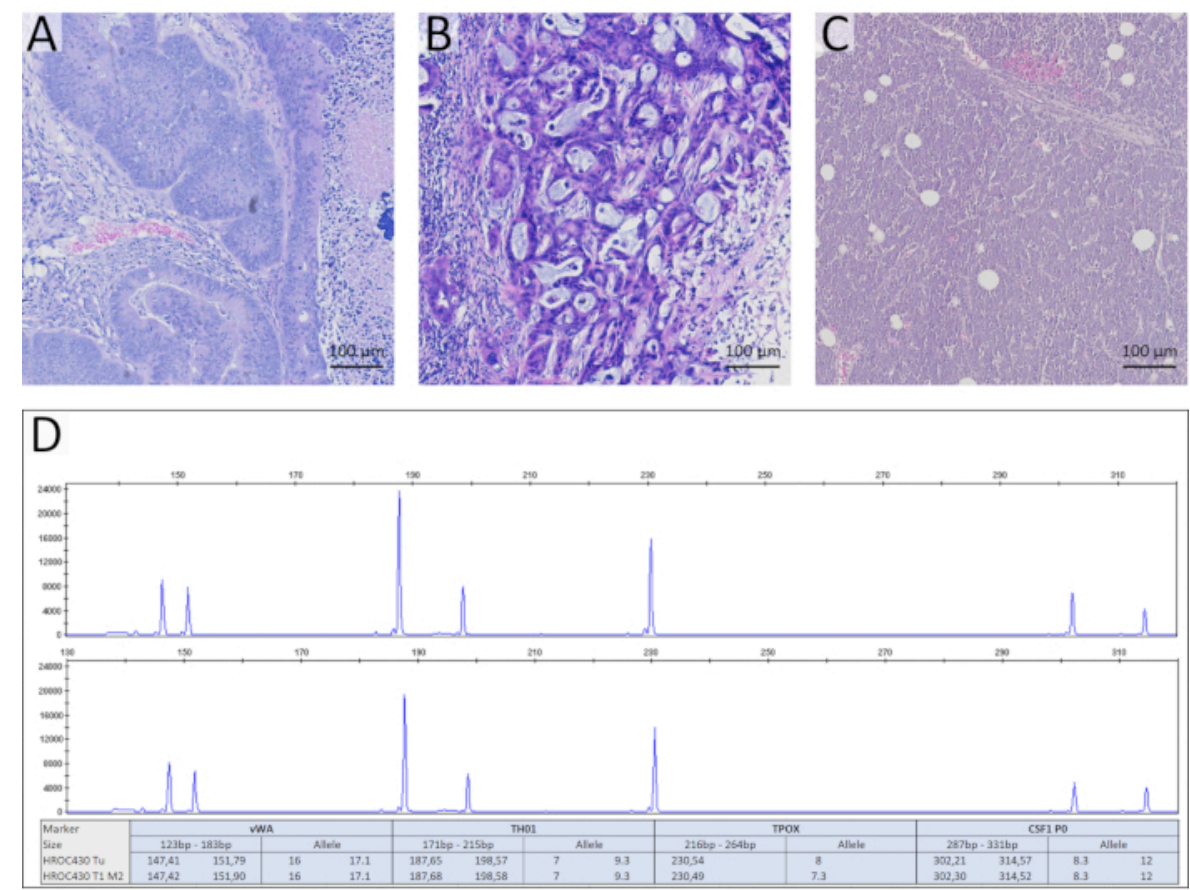

Figure 3: Representative histological comparison of colorectal (A) and pancreatic PDX (B). Human lymphoma at the implantation site mimicking PDX outgrowth (C). Genetic identity testing of a PDX model (HROC430 T1 M2) to the original patient tumor tissue (HROC430Tu) by short tandem repeat (STR) analysis. Comparison of the nine STR loci, vWA, THO1, TPOX, CSF1 PO (FAM dye) and D5S818, D13S317, D7S820, D16S539 (HEX dye) using multiplex PCR with fluorescentlabeled primers following capillary electrophoresis confirmed genetic concordance of the PDX and donor tumor (D). Please click here to view a larger version of this figure.

\section{Discussion}

The generation of a living biobank presupposes, apart from complying with the legal regulations of privacy, medical law and animal welfare, a good infrastructure and a wellcoordinated team. It has proven advantageous to directly involve a part of the surgical staff in the research procedures, since they can very well assess the suitability of the individual patient for tissue donation. Moreover, patients tend to consent with biobanking more frequently, when their written approval is obtained within the course of the surgical informed consent discussion. To save time and resources, cases that will presumably yield insufficient amounts of tumor tissue should not be selected for biobanking. When it comes to specimen acquisition, the maxim "communication is key" is a simple, but often overlooked truth. It only takes a single uninformed 
theatre nurse or surgical colleague to ruin the specimen right at the outset by proceeding as usual and adding formaldehyde to the resection specimen. Therefore, it is absolutely crucial that every single member of the involved staff gets acquainted with the SOP for biobanking. Surgeons should be noticed the day before and right at the start of the procedure about scheduled tissue collection. Furthermore, cases selected for biobanking, should be highlighted in the electronic OR plan. Tissue harvesting from the surgical specimen should be performed by a pathologist. First, this will ensure that the tissue harvesting does not interfere with the final pathological report. Second, this increases the probability of receiving tissue with adequate amounts of viable cancer tissue. Especially in pancreatic cancers with a pronounced desmoplastic reaction and frequent necrotic areas, viable parts are hard to identify macroscopically for the untrained eye. As an exception to this rule, tissue blocks from large hepatic or pulmonal metastases, may at times be excised "back-table" by the surgeon, if surgical margins can be defined macroscopically. Rectal cancer resected by total mesorectal excision (TME), might not be suitable for biobanking, since tissue harvesting from the resected specimen prior to paraffin embedding might interfere with the TME quality assessment. Alternatively, tissue for biobanking can be acquired by transanal biopsy of rectal cancer.

The establishment rates for primary cell cultures derived from the original tumor are generally low. PDX-derived, secondary cell cultures can more likely be successfully established.We recommend testing of different media for each case and use of antibiotic supplements for the first passages to reduce contamination to a minimum since the harvested tissue is rarely sterile. After successful propagation, each individual cell line should be confirmed as a cancer cell line by FACS analysis and regularly tested for mycoplasma contamination.
To exclude cross-contamination, regular STR analysis is advisable. It should be noted, that the establishment protocol for primary and secondary cell lines is constantly subjected to optimization. Details concerning the composition and success rates of the single media are clearly beyond the scope of this work and will be published separately.

For PDX engraftment, tumor tissue can be either implanted directly after resection or cryopreserved in fetal calve serum with $10 \%$ DMSO or similar freezing media for delayed implantation. Implantation immediately upon tumor tissue harvesting puts a strain on logistics and laboratory staff, and xenografting results after cryopreservation are not inferior at all 10. Moreover, incubation of the tissue in Matrigel prior to tumor implantation, significantly increases engraftment rates $^{12}$. We recommend delayed engraftment following definite pathological finding and immediate disposal of erroneously collected tissue specimens. Since the success rate of primary engraftment increases with immunodeficiency of the recipient mouse, we tend to use NSG mice for the very first PDX passage. After the first successful PDX engraftment, $\mathrm{NMRI}^{\mathrm{nu} / \mathrm{nu}}$ mice can and should be used for subsequent passages and tissue expansion. This strain is more robust, cheaper and easier to breed compared to NSG or similar immunodeficient strains, but still shows reasonable engraftment rates. Moreover, its nudeness facilitates implantation and tumor growth monitoring. To increase the engraftment rates in subsequent passages, we recommend direct transfer of freshly harvested PDX tissues to host mice whenever possible, especially for slow growing PDX and cases with a low primary engraftment success rate. Collins and Lang recently reviewed 14 studies of colorectal PDX establishment and reported engraftment rates varying from 14 to $100 \%$ with a median PDX establishment rate of $68 \%$, the latter being consistent with our findings ${ }^{13}$. In 
line with the literature, we observed lower establishment rates of pancreatic compared to colorectal cancer $\mathrm{PDX}^{14}$. Regardless of the host mouse strain and tumor entity, the outgrowth of human, Epstein-Barr Virus (EBV)-associated Bcell lymphomas and murine lymphomas at the implantation side poses an important pitfall ${ }^{15,16}$. If unrecognized, such tumors can "contaminate" subsequent passages and thus confound consecutive results. Unusual fast PDX growth and swelling of cervical, axillar and inguinal lymph nodes are strong indicators of murine lymphoma growth, but regular histological examination of PDX is nevertheless advisable. Furthermore, genetic concordance between PDX and the corresponding donor patient should be tested regularly by STR-analysis. Ideally, the biobank should be linked to a clinical database comprising patients characteristics (general information, survival, relapse free survival, therapy, secondary neoplasia etc.). Due to legal regulations of privacy protection and lack of such an anonymized data base, our clinical data set is regularly administrated and updated manually by the cooperating physicians.

While conventional biobanks are limited to observatory research, a living biobank provides the opportunity for in vitro and in vivo interventions. Patient-derived cell lines are an important tool for fundamental research, high-throughput drug screenings and assessment of new pharmaceutical agents $^{4}$. Corresponding PDX models, however, are of increasing importance, since they closely recapitulate the histology of the original tumor ${ }^{17,18}$ and show a high genetic stability over several passages ${ }^{19,20}$. Our PDX biobank has proven itself as an excellent platform for preclinical and fundamental research ${ }^{6,21}$. Moreover, since large PDX collections adequately reflect the inter-individual heterogeneity of the patient population, the PDX clinical trial (PCT) approach (one animal per model per treatment) has gained importance for drug development since it allows the faithful prediction of clinical response to new drugs and combinatorial regimen ${ }^{8}$. We also are currently evaluating new experimental drugs in small PCT trials.

Despite these promising results, the median establishment duration of 12.2 month, impedes the clinical applicability of PDX models as "avatar mice" for testing anticancer treatment options, at least for those patients in need of immediate adjuvant or even neoadjuvant treatment ${ }^{22}$. An additional disadvantage of standard PDX models is the lack of usability for immunotherapy testing due to host mice's immunodeficiency. To overcome these limitations, several "humanized" mouse strains have been developed. These mice are heavily immunocompromised, but can be reconstituted with various types of human bone marrowderived cells or $\mathrm{CD} 4^{+}$hematopoietic stem cells subsequent to PDX outgrowth ${ }^{23}$, allowing the evaluation of lymphocytemediated cytotoxicity and of therapy response to immune checkpoint inhibitor treatment ${ }^{24,25}$.

In recent years, patient-derived organoids (PDO) emerged as important cancer models competing with PDX. Derived from intact tumor pieces and cultured in an extracellular matrix scaffold, these three-dimensional structures closely reflect the histologic and genetic properties of the original tumor. The possibility of long-term expansion and cryopreservation renders PDO an ideal supplement of a living biobank ${ }^{26,27}$. In addition to a relatively high establishment rate, reliable drug response prediction has been reported for PDO of several tumor entities ${ }^{28}$. Moreover, PDOs have even been generated from circulating tumor cells and also the simultaneous establishment of organoids from corresponding healthy tissue is possible, allowing assessment of therapy-related toxicity on a patient-individual basis ${ }^{29,30}$. However, compared to 
conventional 2D cell cultures, organoid culture is time and resource consuming and artificial extracellular matrix compounds can interfere with certain analytic procedures ${ }^{31}$. Moreover, cancer organoids are susceptible to overgrowth by faster growing, non-malignant organoids derived from healthy epithelium $^{30}$. Due to a lack of stroma, blood vessels and immune cells, PDOs are mostly inapplicable for the testing of antiangiogenic immunotherapeutic agents. Yet, new culturing methods allow the modeling of tumor microenvironment in vitro, rendering PDOs a true contender for PDX models ${ }^{32}$. In the near future, patient-individual tumor models, combined with powerful genetic tools like next-generation sequencing, will hopefully pave the path to true precision medicine and tailored-treatment approaches.

\section{Disclosures}

None.

\section{Acknowledgments}

We kindly acknowledge Jenny Burmeister, our graphical assistant, for the recording and editing of the video. Furthermore, we thank our colleagues of the surgical and pathological department for the longstanding collaboration. We would also like to thank Marcus Müller, production manager of the IT and Media Centre, University of Rostock, for supplying the audio recording equipment and refining the sound quality.

FUNDING: The German Cancer Aid Foundation (DKH e.V.), grant number 108446, and grant number TBI-V-1-241VBW-084 from the state Mecklenburg-Vorpommern partly funded this research.

\section{References}

1. Sartore-Bianchi, A. et al. PIK3CA mutations in colorectal cancer are associated with clinical resistance to EGFRtargeted monoclonal antibodies. Cancer research. 69 (5), 1851-1857, (2009).

2. Pauli, C. et al. Personalized In Vitro and In Vivo Cancer Models to Guide Precision Medicine. Cancer discovery. 7 (5), 462-477, (2017).

3. Lipson, D. et al. Identification of new ALK and RET gene fusions from colorectal and lung cancer biopsies. Nature medicine. 18 (3), 382-384, (2012).

4. Wilding, J.L., Bodmer, W.F. Cancer cell lines for drug discovery and development. Cancer research. 74 (9), 2377-2384, (2014).

5. Mouradov, D. et al. Colorectal cancer cell lines are representative models of the main molecular subtypes of primary cancer. Cancer research. 74 (12), 3238-3247, (2014).

6. Klier, U., Maletzki, C., Kreikemeyer, B., Klar, E., Linnebacher, M. Combining bacterial-immunotherapy with therapeutic antibodies: a novel therapeutic concept. Vaccine. 30 (17), 2786-2794, (2012).

7. DiMasi, J.A., Reichert, J.M., Feldman, L., Malins, A. Clinical approval success rates for investigational cancer drugs. Clinical pharmacology and therapeutics. 94 (3), 329-335, (2013).

8. Gao, H. et al. High-throughput screening using patientderived tumor xenografts to predict clinical trial drug response. Nature medicine. 21 (11), 1318-1325, (2015).

9. Mullins, C.S. et al. Integrated Biobanking and Tumor Model Establishment of Human Colorectal Carcinoma Provides Excellent Tools for Preclinical Research. Cancers. 11 (10), (2019). 
10. Kuehn, F. et al. Establishment and characterization of HROC69 - a Crohn's related colonic carcinoma cell line and its matched patient-derived xenograft. Scientific reports. 6, 24671, (2016).

11. Dangles-Marie, V. et al. Establishment of human colon cancer cell lines from fresh tumors versus xenografts: comparison of success rate and cell line features. Cancer research. 67 (1), 398-407, (2007).

12. Gock, M. et al. Tumor Take Rate Optimization for Colorectal Carcinoma Patient-Derived Xenograft Models. BioMed research international. 2016, 1715053, (2016).

13. Collins, A.T., Lang, S.H. A systematic review of the validity of patient derived xenograft (PDX) models: the implications for translational research and personalised medicine. PeerJ. 6, e5981, (2018).

14. Brown, K.M. et al. Patient-derived xenograft models of colorectal cancer in pre-clinical research: a systematic review. Oncotarget. 7 (40), 66212-66225, (2016).

15. Zhang, L. et al. The extent of inflammatory infiltration in primary cancer tissues is associated with lymphomagenesis in immunodeficient mice. Scientific reports. 5, (2015).

16. Moyer, A.M. et al. Spontaneous murine tumors in the development of patient-derived xenografts: a potential pitfall. Oncotarget. 10 (39), 3924-3930, (2019).

17. Prall, F., Maletzki, C., Hühns, M., Krohn, M., Linnebacher, M. Colorectal carcinoma tumour budding and podia formation in the xenograft microenvironment. PloS one. 12 (10), e0186271, (2017).

18. Guenot, D. et al. Primary tumour genetic alterations and intra-tumoral heterogeneity are maintained in xenografts of human colon cancers showing chromosome instability. The Journal of pathology. 208 (5), 643-652, (2006).

19. Mattie, M. et al. Molecular characterization of patientderived human pancreatic tumor xenograft models for preclinical and translational development of cancer therapeutics. Neoplasia (New York, N.Y.). 15 (10), 1138-1150, (2013).

20. Cho, Y.B. et al. Colorectal cancer patient-derived xenografted tumors maintain characteristic features of the original tumors. The Journal of surgical research. 187 (2), 502-509, (2014).

21. Maletzki, C. et al. Functional Characterization and Drug Response of Freshly Established Patient-Derived Tumor Models with CpG Island Methylator Phenotype. PloS one. 10 (11), e0143194, (2015).

22. Katsiampoura, A. et al. Modeling of Patient-Derived Xenografts in Colorectal Cancer. Molecular cancer therapeutics. 16 (7), 1435-1442, (2017).

23. Wege, A.K. et al. Humanized tumor mice--a new model to study and manipulate the immune response in advanced cancer therapy. International journal of cancer. 129 (9), 2194-2206, (2011).

24. Herndler-Brandstetter, D. et al. Humanized mouse model supports development, function, and tissue residency of human natural killer cells. Proceedings of the National Academy of Sciences of the United States of America. 114 (45), E9626-E9634, (2017).

25. Capasso, A. et al. Characterization of immune responses to anti-PD-1 mono and combination immunotherapy in hematopoietic humanized mice implanted with tumor xenografts. Journal for immunotherapy of cancer. 7 (1), $37,(2019)$. 
26. Sato, T. et al. Long-term expansion of epithelial organoids from human colon, adenoma, adenocarcinoma, and Barrett's epithelium. Gastroenterology. 141 (5), 1762-1772, (2011).

27. Clevers, H. Modeling Development and Disease with Organoids. Cell. 165 (7), 1586-1597, (2016).

28. Vlachogiannis, G. et al. Patient-derived organoids model treatment response of metastatic gastrointestinal cancers. Science (New York, N.Y.). 359 (6378), 920-926, (2018).

29. Gao, D. et al. Organoid cultures derived from patients with advanced prostate cancer. Cell. 159 (1), 176-187, (2014).

30. Drost, J., Clevers, H. Organoids in cancer research. Nature reviews. Cancer. 18 (7), 407-418, (2018).

31. Abe, Y. et al. Improved phosphoproteomic analysis for phosphosignaling and active-kinome profiling in Matrigelembedded spheroids and patient-derived organoids. Scientific reports. 8 (1), 11401, (2018).

32. Neal, J.T. et al. Organoid Modeling of the Tumor Immune Microenvironment. Cell. 175 (7), 1972-1988.e16, (2018). 